\title{
Clinical study on postoperative recurrence in patients with pNO esophageal squamous cell carcinoma
}

\author{
Xu-feng Guo ${ }^{\dagger}$, Teng Mao ${ }^{\dagger}$, Zhi-tao Gu, Chun-yu Ji, Wen-tao Fang ${ }^{*}$ and Wen-hu Chen
}

\begin{abstract}
Background: Despite increasingly radical surgery for esophageal carcinoma, many patients still develop tumor recurrence after operation. This study was designed to analyze the clinical and pathologic influencing factors of early recurrence in patients with histological node-negative (pNo stage) esophageal squamous cell carcinoma (ESCC) after radical esophagectomy.
\end{abstract}

Methods: A retrospective study on 112 consecutive pNO stage ESCC patients who underwent esophagectomy with lymphadenectomy by the same surgical team from January 2004 to December 2010. There were 92 male and 20 female patients, aging from 36 to 80 years with a mean age of 60.3 years. The Cox proportional hazards model was used to determine the independent risk factors for recurrence within 3 years after the operation.

Results: Recurrence was recognized in 45 patients (40.2\%) within 3 years after operation. The median time to tumor recurrence was 17.4 months. Locoregional recurrence was found in 38 patients (33.9\%) and hematogenous metastasis in 7 patients (6.3\%). However, locoregional recurrence accounted for $84.4 \%$ of all relapse patients. Recurrence closely correlated with tumor location, grade of differentiation, primary tumor stage (pT) and pathologic stage $\left(x^{2}=6.380\right.$ to $\left.18.837, p<0.05\right)$. The Cox multivariate analysis showed that upper/middle thoracic location $(\mathrm{OR}=1.092, p=0.049)$ and $\mathrm{pT3}-4 \mathrm{a}$ stage $(\mathrm{OR}=3.296, p=0.017)$ were independent risk factors for postoperative locoregional recurrence.

Conclusion: Locoregional recurrence was the most common recurrence pattern of patients with pNO ESCC within 3 years after operation. Upper/middle thoracic location and pT3-4a stage were independent risk factors for locoregional recurrence of pNO ESCC after radical esophagectomy.

Keywords: Esophagus neoplasms, Lymph node dissection, Recurrence and metastasis

\section{Background}

In China, the predominant pathologic type of esophageal carcinoma is squamous cell carcinoma (ESCC), and many tumors locate in the middle thoracic. The overall 5 -year survival rate of patients with lymph node metastasis (LNM) after surgical resection was only about $20 \%$ [1], and most of these patients died of tumor relapse. Recently, the therapeutic efficacy for ESCC had been greatly improved due to the establishment and promotion

\footnotetext{
*Correspondence: vwtfang@hotmail.com

${ }^{\dagger}$ Equal contributors

Department of Thoracic Surgery, Shanghai Chest Hospital, School of Medicine, Shanghai Jiaotong University, 241 West Huaihai Road, Shanghai
} 200030, China

\section{Biomed Central}

of multidisciplinary treatment model [2]. However, even for histological node-negative (pN0 stage) ESCC patients, a large number of these patients still experienced recurrence after operation [3]. So it was important to study the causes and characteristics of recurrence when designing therapeutic strategies, including operative procedures and combined therapies.

The purposes of this study were to explore the pattern and time of recurrence after extended radical esophagectomy with systemic lymphadenectomy, to identify the prognostic factors responsible for recurrence, and to discuss therapeutic strategies that might be helpful to decrease recurrence and improve survival. 


\section{Methods \\ Patients}

This study was approved by the Research Ethics Committee of Shanghai Jiao Tong University School of Medicine and all participants at the Shanghai Chest Hospital gave written informed consent. A total of 112 consecutive pN0 stage ESCC patients who underwent esophagectomy with lymphadenectomy by the same surgical team from January 2004 to December 2010. The inclusion criteria were as follows: (1) routine preoperative esophageal endoscopy, a clear pathology of squamous cell carcinoma, (2) assessment of tumor location by the upper gastrointestinal barium swallow, (3) no evidence of tumescent cervical or supraclavicular lymph node disease was noted on physical examination, and a preoperative computed tomograpy (CT) scan or cervical B ultrasound study indicated no cervical or supraclavicular lymph node metastasis, (4) positron emission tomography to rule out distant metastasis, (5) no preoperative radiotherapy and/or chemotherapy, (6) pathologically confirmed lymph node-negative after operation, (7) all the patients were R0 resection of pathologically confirmed, (8) all the patients did not receive any adjuvant therapy before recurrence. Of all the patients, there were 92 men and 20 women, and the median age of 60.3 years (range: $36-80$ years). The tumor location and the TNM classification were determined according to criteria established by the Union for International Cancer Control (UICC) in 2009 [4].

\section{Surgical procedure}

At operation the patient was placed in the $90^{\circ}$ left lateral decubitus position. After a right posterolateral thoracotomy, the chest was entered through the fifth intercostal space. The azygos vein arch was divided, and the esophagus was dissected from the esophagogastric junction to the apex of the chest. When the tumor invasion obviously extended outside the esophagus, the thoracic duct was routinely ligated above the diaphragm. An upper midline abdominal incision was also made, and the abdomen was explored. During mobilization of the stomach, care was taken to preserve the right gastroepiploic vessels and arcades. The left gastric artery and vein were isolated and doubly ligated at their origin. The hiatus was enlarged and the stomach was pulled into the chest. An end-to-side esophagogastric anastomosis was performed within the apex of the chest (above the azygos vein) and the stomach was secured into the mediastinum (Ivor-Lewis).When the tumor located in upper thoracic, we will pull the stomach to neck by post-sternum tunnel for anastomosis (McKeown).

According to a lymph node mapping system for esophageal cancer (Japanese esophageal oncology group, JEOG) (Figure 1), thoraco-abdominal 2-field lymphadenectomy was undertaken. Some patients were undertaken cervicothoraco-abdominal 3-field lymphadenectomy based on the positive results of preoperative cervical ultrasonography. The fields of lymph node dissections were as follows: 100, superficial cervical lymph nodes; 101, cervical esophageal lymph nodes; 102, deep cervical lymph nodes; 104, Supraclavicular lymph nodes; 105, upper thoracic esophageal lymph nodes; 106, recurrent laryngeal nerve lymph nodes; 107, subcarinal lymph nodes; 108, middle thoracic esophageal lymph nodes; 109, hilar lymph nodes; 110, lower thoracic esophageal lymph nodes; 111, diaphragmatic lymph nodes; 1 , cardial lymph nodes (right); 2, cardial lymph nodes (left); 3 , lesser curvature lymph nodes; 7 , left gastric lymph nodes.

\section{Follow-up examinations}

Patients were routinely accepted the first examination at 3-4 weeks after the operation, examined at 3-month intervals for 1 year and at 6-month intervals thereafter. During each follow-up visit, the patient underwent a clinical evaluation, blood biochemistry examination including tumor markers, and chest radiography. Endoscopy, ultrasonography (US) of the neck and abdomen, and computed tomography (CT) of the neck, thorax, and abdomen were performed at least once a year. More selective investigations such as positron emission tomography (PET), bone scintigraphy, and Head-enhanced magnetic resonance imaging (MRI) were carried out based on specific symptomatology, clinical examination, and biochemical profile. Detection of a suspected recurrence at any one site was followed by a thorough detailed investigation to confirm or refute the occurrence and to examine every other site.

\section{Definition of locoregional and hematogenous recurrence}

The first recurrence was noted, and any additional recurrence found within one month was considered to have occurred simultaneously. These lesions were classified as locoregional (at the remaining esophagus, the anastomotic site, or the mediastinum, cervical, supraclavicular and celiac axis lymph nodes) and hematogenous (in the distant organs such as liver, lung, bone and pleura, peritoneum) recurrence. Simultaneous locoregional and hematogenous recurrence was classified as a hematogenous recurrence.

\section{Statistical analysis}

The SPSS 16.0 software package was used for data analysis. Data were expressed as median with ranges (minimummaximum) or as percentages. The chi-square test was used to evaluate differences in clinicopathologic features. The Cox proportional hazards model was used to determine 


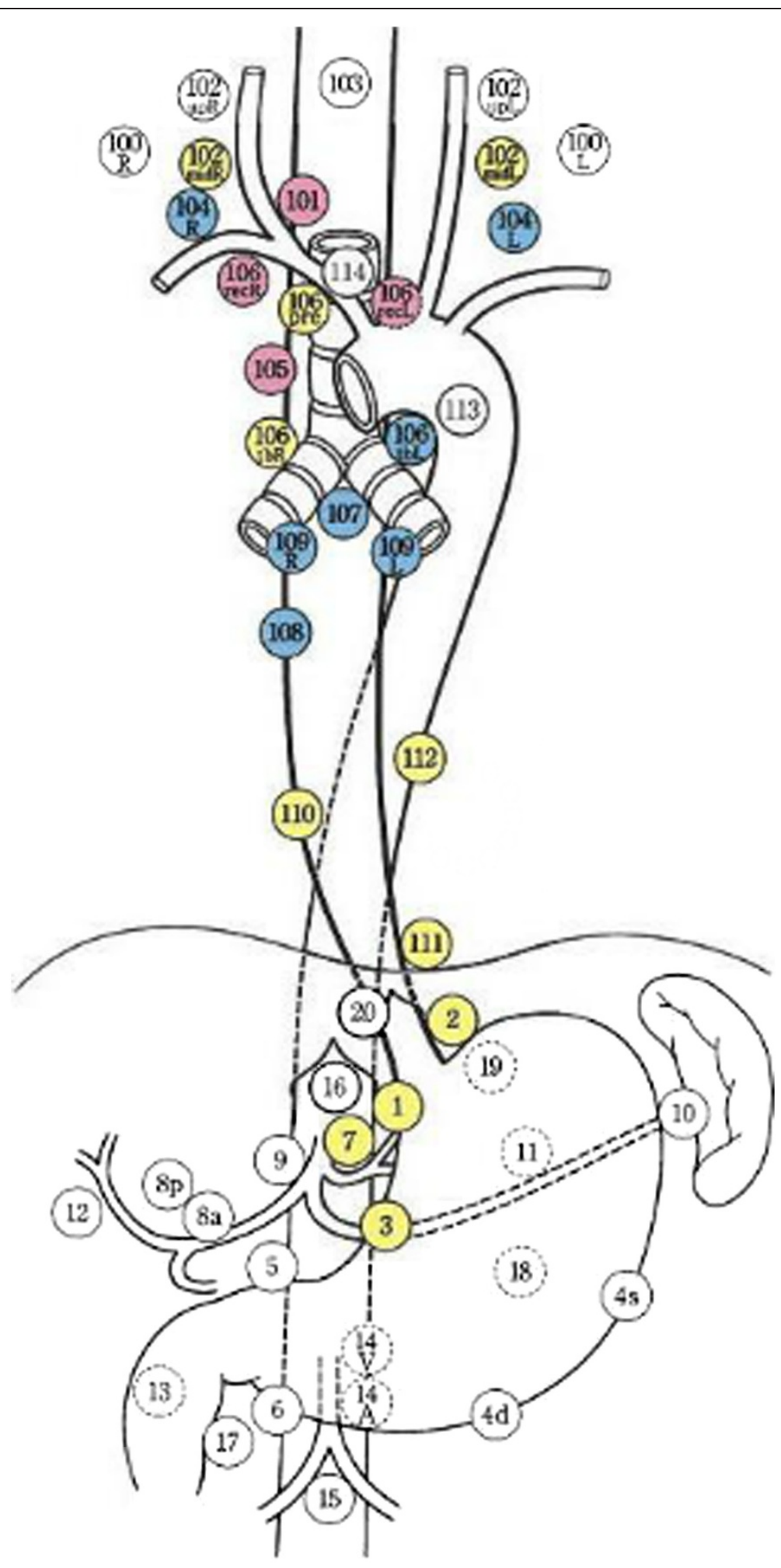

Figure 1 Regional lymph node stations for staging esophageal cancer (JEOG).

the independent risk factors for recurrence within 3 years after the operation. Estimation of recurrence was calculated with the Kaplan-Meier method, and the statistical differences were analyzed with the log-rank test. A $p$ value of $<0.05$ was considered statistically significant for all procedures. 


\section{Results}

112 patients were investigated and recurrence was recognized in 45 patients (40.2\%) in the first 3 years after operation. The median disease-free interval until recurrence was 17.4 months (range: 3.5-26.8 months). Of these, 39 were men and 6 were women, and the median age was 56 years (range: $39-76$ years).

\section{Pattern of recurrence}

38 patients (33.9\%) developed a locoregional recurrence; 7 patients $(6.3 \%)$ developed a hematogenous recurrence, including 2 patients (1.8\%) with simultaneous locoregional and hematogenous recurrence. However, locoregional recurrence accounted for $84.4 \%$ of all relapse patients. The relationship between clinicopathologic factors and recurrent disease was depicted in Table 1 . There were significant

Table 1 Clinical data of 112 pNO ESCC patients undergoing radical esophagectomy with lymphadenectomy

\begin{tabular}{|c|c|c|c|c|}
\hline \multirow[t]{2}{*}{ Variables } & \multicolumn{2}{|c|}{ Recurrence } & \multirow[t]{2}{*}{ Statistic } & \multirow[t]{2}{*}{$p$ Value } \\
\hline & Positive & Negtive & & \\
\hline No. of patients & 45 & 67 & - & - \\
\hline Gender (M/F) & $39 / 6$ & $53 / 14$ & $x^{2}=0.497$ & 0.173 \\
\hline Age (years) & $60 \pm 5$ & $61 \pm 7$ & $t=4.877$ & 0.782 \\
\hline Tumor location & & & $x^{2}=7.845$ & 0.038 \\
\hline Upper & 11 & 10 & & \\
\hline Middle & 29 & 35 & & \\
\hline Lower & 5 & 22 & & \\
\hline Grade of differentiation & & & $x^{2}=6.380$ & 0.043 \\
\hline Well (G1) & 4 & 12 & & \\
\hline Moderately (G2) & 9 & 22 & & \\
\hline Poorly (G3) & 32 & 33 & & \\
\hline pT status & & & $x^{2}=18.837$ & 0.006 \\
\hline $\mathrm{T} 1$ & 0 & 19 & & \\
\hline $\mathrm{T} 2$ & 6 & 19 & & \\
\hline T3 & 35 & 26 & & \\
\hline T4a & 4 & 3 & & \\
\hline Pathological staging & & & $x^{2}=7.845$ & 0.024 \\
\hline Stage I & 1 & 20 & & \\
\hline Stage ॥ & 9 & 18 & & \\
\hline Stage III & 31 & 26 & & \\
\hline Stage IIIA & 4 & 3 & & \\
\hline Lymphadenectomy & & & $x^{2}=5.226$ & 0.119 \\
\hline Two field & 39 & 58 & & \\
\hline Three field & 6 & 9 & & \\
\hline Intralmural metastasis & & & $x^{2}=3.593$ & 0.336 \\
\hline Positive & 4 & 0 & & \\
\hline Negtive & 41 & 67 & & \\
\hline
\end{tabular}

differences in tumor location $(p=0.038)$, grade of differentiation $(p=0.043)$, primary tumor stage $(\mathrm{pT})(p=0.006)$ and pathologic stage $(p=0.024)$ between patients with recurrence and those without recurrence.

\section{The distribution of the sites of tumor recurrence}

With respect to the locoregional tumor recurrence, 21 patients (15.3\%) had recurrence in only cervical/ supraclavicular lymph nodes, 13 patients $(34.2 \%)$ had recurrence in only mediastinal lymph nodes, 6 (15.8\%) in only abdominal lymph nodes. In cases of hematogenous recurrence, the main sites of recurrence were liver (6/7), lung (2/7), and bone (1/7). There was no one had recurrence in brain, pleura and omentum in our patients within 3 years after operation. Lymph node metastasis occured earlier than hematogenous recurrence (Table 2 and Figure 2).

The multivariate analysis for locoregional recurrence The multivariate analysis by the Cox proportional hazards model demonstrated that Upper/middle thoracic location $(p=0.049, \mathrm{OR}=1.092)$ and $\mathrm{pT} 3-4 \mathrm{a}$ stage $(p=0.017$, $\mathrm{OR}=3.296$ ) were significant risk factors for locoregional recurrence of pNO ESCC after operation (Table 3). We did not find a significant correlation between locoregional recurrence and gender, histological differentiation, or lymphadenectomy style.

The recurrence curve in pN0 patients with different pT stage was depicted in Figure 3 The log-rank test showed that there was a significant difference among the recurrence rates of these two groups $(p=0.000)$. The

Table 2 Site of locoregional and hematogenous recurrence in $\mathbf{4 5}$ patients

\begin{tabular}{|c|c|c|c|}
\hline Site of recurrence & No. & Percent (\%) & $\begin{array}{l}\text { Time of recurrence } \\
\text { (month) }\end{array}$ \\
\hline Locoregional recurrence $^{1}$ & 38 & & \\
\hline Cervical/supraclavicular node & 21 & 55.3 & $14.9(3.5 \sim 17.7)$ \\
\hline Mediastinal node & 13 & 34.2 & \\
\hline upper & 9 & 23.7 & $17.2(4.8 \sim 23.2)$ \\
\hline middle & 3 & 7.9 & $19.2(7.1 \sim 25.3)$ \\
\hline lower & 1 & 2.6 & 23.1 \\
\hline Abdominal node & 6 & 15.8 & $18.4(4.5 \sim 26.8)$ \\
\hline Anastomotic & 1 & 2.6 & 8.6 \\
\hline Hematogenous recurrence $^{2}$ & 7 & & \\
\hline Liver & 6 & 85.7 & $10.4(5.2 \sim 20.4)$ \\
\hline Lung & 2 & 28.6 & $12.8(9.4 \sim 16.2)$ \\
\hline Bone & 1 & 14.3 & 18.5 \\
\hline Others(brain/pleura/omentum) & 0 & & \\
\hline
\end{tabular}




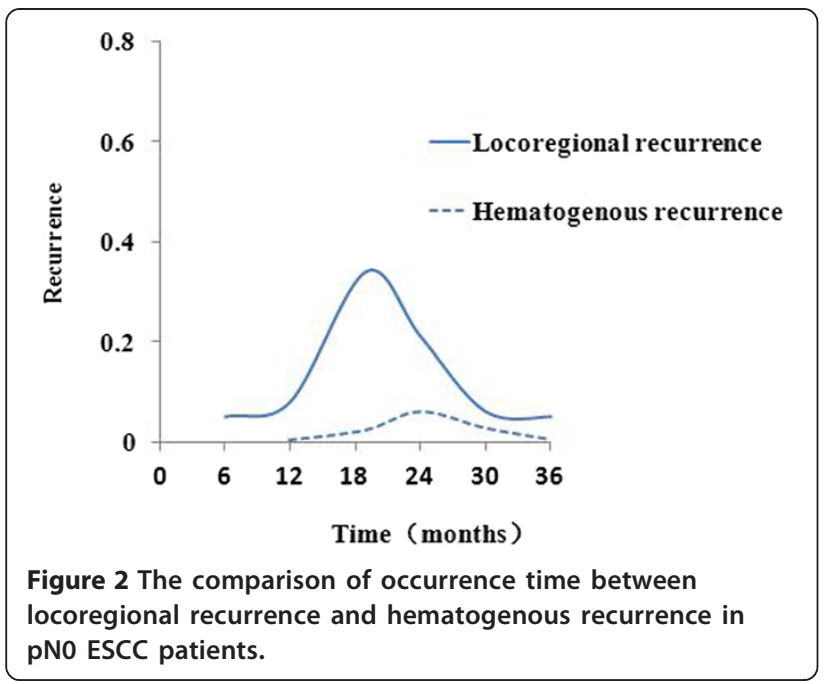

recurrence rate of the patients with upper/middle thoracic location was higher than the rate of lower thoracic location $(p=0.045)$ (Figure 4).

\section{Discussion}

The 7th edition UICC TNM staging of esophageal cancer (2009) increased cell differentiation (G) and histological type $(\mathrm{H})$ as a staging reference factor, also the pT3 stage (tumor invades adventitia) and pT4a stage (resectable tumor invading pleura, pericardium, or diaphragm) were distinguished [4]. With the wide application of the new version of the standard staging for esophageal cancer, the latest esophageal cancer treatment guidelines by National Comprehensive Cancer Network (NCCN) recommended for pNO ESCC patients [5]: (1) pT1-4aN0M0 patients with ESCC, follow-up and observation after operation; (2) pT1-2N0M0 patients with adenocarcinoma, recommended follow-up and observation. However, some of the pT2NOM0 patients with high risk (poorly differentiated, lymphatic invasion, perineural invasion and $<50$ years of age), recommended postoperative chemoradiation; (3) pT3-4aNOM0 patients with adenocarcinoma were recommended postoperative chemoradiation or follow-up.
As we can see, prophylactic postoperative adjuvant therapy was not recommended for pT1-2N0M0 patients. However, there was no uniform international standards on whether pT3-4aNOM0 patients need postoperative adjuvant therapy or not, and what specific treatment should be chosen currently. It is worth noting that China, as a country with high incidence of esophageal cancer, nearly all of patients are squamous cell carcinoma. It is obviously different with the western countries in which the main histology of esophageal cancer was adenocarcinoma. Meanwhile, ESCC occur mostly in upper and middle thoracic, but adenocarcinoma occur mostly in lower thoracic. Whether from the perspective of anatomical or histological analysis, ESCC is more easy to have mediastinal lymph node metastasis even the neck and peritoneal metastasis than adenocarcinoma. Seen in this light, the foreign experience might not be suitable for China. Therefore, to study the risk factors for recurrence and metastasis in pNo patients with ESCC, following suitable adjuvant treatment had important clinical significance for reducing recurrence and improving long-term survival.

Our cases showed that there was $40.2 \%$ of 112 pN0 ESCC patients had recurrence within 3 years after operation, with a median time (17.4 months) to recurrence, similar to the results reported in the literature [6]. Univariate analysis showed that recurrence closely correlated with tumor location, grade of differentiation, primary tumor stage (pT) and pathologic stage. The COX proportional hazards regression multivariate analysis confirmed that upper/middle thoracic location and pT3-4a stage were independent risk factors for postoperative locoregional recurrence. The $\mathrm{pT}$ stage (Wald $=22.731 ; \mathrm{OR}=3.296$ ) was the most important risk factor on recurrence and metastasis of pNO ESCC according to the Wald value. With increasing $\mathrm{pT}$ stage, the chance of recurrence and metastasis in pNO esophageal cancer patients also increased significantly. The reason was that esophagus lacks serosa, once the tumor invades adventitia, not only the submucosal longitudinal lymphatic vessels but also lateral mediastinal lymphatic vessels originating from the muscle layer contribute greatly to the LNM [7]. In present study, the

Table 3 The multivariate analysis for locoregional recurrence of pNO ESCC

\begin{tabular}{|c|c|c|c|c|c|c|}
\hline Variable & B & SE & Wald & $p$ Value & OR & $95 \% \mathrm{Cl}$ \\
\hline \multicolumn{7}{|l|}{ Tumor location } \\
\hline (upper/middle versus lower) & 1.087 & 0.557 & 3.808 & 0.049 & 1.092 & $1.088 \sim 2.383$ \\
\hline \multicolumn{7}{|l|}{ Grade of differentiation } \\
\hline$(\mathrm{G} 3$ versus $\mathrm{G} 1+2)$ & 0.478 & 0.235 & 4.143 & 0.068 & 1.613 & $0.690 \sim 1.551$ \\
\hline \multicolumn{7}{|l|}{ Depth of invasion } \\
\hline (pT3-4a versus pT1-2) & 1.193 & 0.250 & 22.731 & 0.017 & 3.296 & $2.019 \sim 5.381$ \\
\hline
\end{tabular}

B: regression coefficient; SE: standard error; Wald: wald value; OR: odds ratio; Cl: confidence interval. 


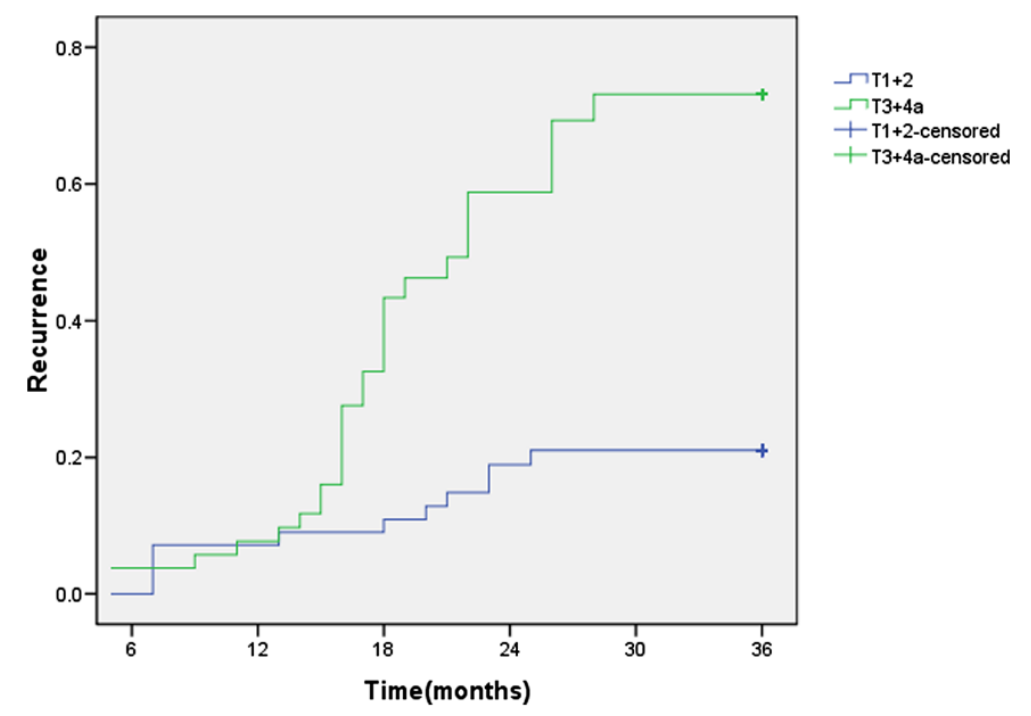

Figure 3 Recurrence curve for the patients with different pT stage.

poorly differentiation (G3) did not have statistical significance with tumor recurrence might be due to small number of cases, but the $p$ value (0.068) was close to 0.05 . In addition, four patients with pathologically confirmed the presence of intralmural metastasis, three of them had cervical lymph node recurrence 2 years after operation and another appeared residual esophageal tumor recurrence 1 year after operation. It was suggesting that intralmural metastasis might be an important risk factor for recurrence of $\mathrm{pNO}$ ESCC. Therefore, taking into account the possibility of multi-center origin in esophageal cancer and intralmural metastasis, we have to do the following in operation: (1) resection with sufficient length of esophagus; (2) complete resection of the lesion and the surrounding involved structures; (3) radical elimination of lymphatic tissue.

In the present study, the most common recurrence pattern of pNO patients with ESCC after operation was locoregional recurrence $(38 / 45,84.4 \%)$ and LNM (37/38, $97.4 \%$ ) was the main form, mainly in the cervicothoracic junction. Therefore, the cervicothoracic junction was the key position for preventing recurrence of pN0 patients with ESCC after operation. In addition, the difference of recurrence between two-field (thoracic and abdominal) lymph node dissections and three-field (cervical, thoracic and abdominal) lymph node dissections was not significant in our study. However, the literature had reported that three-field lymph node dissections could reduce

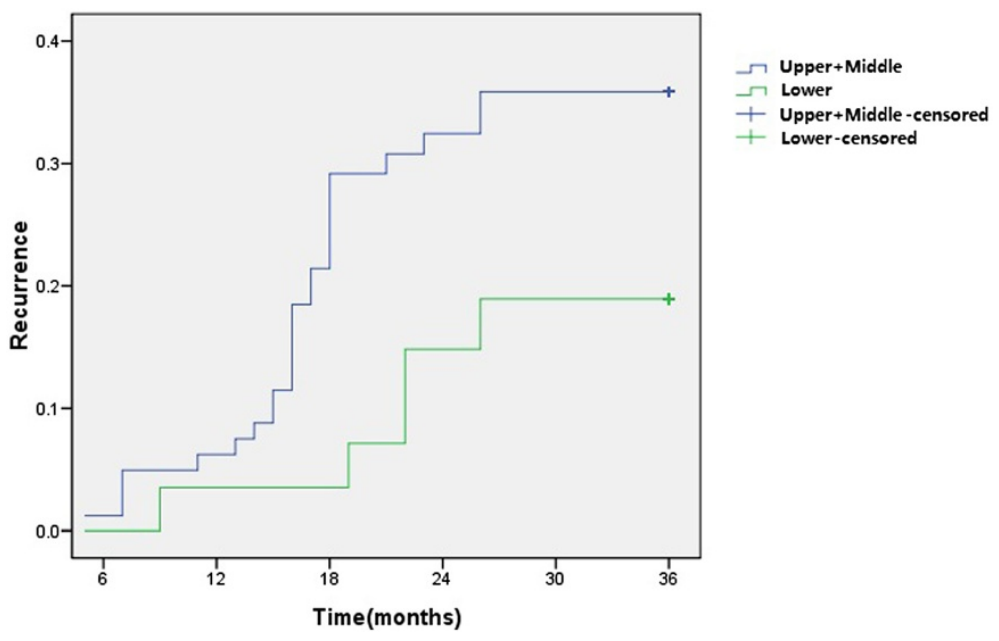

Figure 4 Recurrence curve for the patients with different tumor location. 
recurrence and metastasis rate significantly [8]. The possible explanations were: Firstly, patients with pNO ESCC might not need the three-field lymph node dissections; Secondly, there were statistical deviation due to small number of patients were given three-field lymph node dissections in this group, and this study was a retrospective analysis. Cervical B ultrasound was helpful to the diagnosis of LNM in cervicothoracic junction [9], combined with preoperative CT scan of chest/abdomen and positron emission tomography, establishing an accurate clinical staging was helpful to select the patients who do need three-field lymph node dissections [10]. We also took into account: (1) there were many important organs, large vessels in cervicothoracic junction and the region of recurrent laryngeal nerve was a relatively difficult position for systemic lymph node dissections because of higher requirements of surgical techniques; (2) three-field lymph node dissections had to face the bottleneck which can not further improve the long-term survival obviously. So, the postoperative radiotherapy and/or chemotherapy might be a good alternative.

Our data did not find independent risk factors for $\mathrm{pNO}$ ESCC patients with hematogenous metastasis, suggesting that hematogenous metastasis was mostly influenced by the biological behavior of tumor cell rather than depth of tumor invasion, LNM and differentiation [11]. Thus, it was necessary to carry out prospective clinical study for evaluating whether vascular invasion, lymphatic invasion, and perineural invasion and so on were related with hematogenous metastasis or not.

\section{Conclusions}

In summary, we have confirmed that locoregional recurrence was the most common recurrence pattern of patients with pNO ESCC within 3 years after operation. Upper/middle thoracic location and pT3-4a stage were independent risk factors for locoregional recurrence of pNO ESCC after radical esophagectomy. For these highrisk groups, prophylactic radiotherapy for bilateral supraclavicular and upper mediastinal might be helpful to reduce the recurrence rate.

\section{Abbreviations \\ pNO stage: Histological node-negative; ESCC: Esophageal squamous cell carcinoma; LNM: Lymph node metastasis; US: Ultrasonography; CT: Computed tomography; PET: Positron emission tomography; MRI: Magnetic resonance imaging.}

\section{Competing interests}

The authors declare that they have no competing interest.

\section{Authors' contributions}

Design the experiments: XFG, TM, WTF. Do and interpret the experiments: XFG, ZTG, CYJ, WHC. Write the manuscript: XFG. All authors read and approved the final manuscript. Thanks very much for your efforts and vable time.
Received: 10 June 2014 Accepted: 18 August 2014

Published: 28 August 2014

\section{References}

1. Chen W, He Y, Zheng R, Zhang S, Zeng H, Zou X, He J: Esophageal cancer incidence and mortality in China, 2009. J Thorac Dis 2013, 5:19-26.

2. Mulligan CR Jr: Multidisciplinary management of esophageal cancer. Surg Oncol Clin N Am 2013, 22:217-246.

3. Li SH, Wang Z, Liu XY, Liu FY, Sun ZY, Xue H: Lymph node micrometastasis: a predictor of early tumor relapse after complete resection of histologically node-negative esophageal caner. Surg Today 2007, 37:1047-1052.

4. Edge SB, Byrd DR, Compton CC, Fritz AG, Greene FL, Trotti A: AJCC Cancer Staging Manual. 7th edition. New York: Springer; 2009.

5. NCCN practice guidelines in oncology, esophageal and esophagogastric junction cancers. (Version.2.2013). [EB/OL]. [2013-02-21]. http//wwww.nccn.org.

6. Zi-jiang Z, Xue-zhong C, Niu R, Xue JJ, Chen J, Zheng C: Risk factors for recurrence or metastasis of tumor in patients with esophageal carcinoma after esophagectomy. Chin I Clin Thorac Cardiovasc Surg 2011, 18:521-524

7. Tachimori $Y$, Nagai $Y$, Kanamori N, Hokamura N, Igaki H: Pattern of lymph node metastases of esophageal squamous cell carcinoma based on the anatomical lymphatic drainage system. Dis Esophagus 2011, 24:33-38.

8. Shim YM, Kim HK, Kim K: Comparison of survival and recurrence pattern between two-field and three-field lymph node dissections for upper thoracic esophageal squamous cell carcinoma. J Thorac Oncol 2010, 5:707-712

9. Fang Wen $\mathrm{T}$, Zhang Zhan $\mathrm{H}$, Chen Wen $\mathrm{H}$, Jiang $\mathrm{Y}$, Tao Ju W, Zhong $Z \mathrm{y}$ : Ultrasound surveillance of cervical lymph node metastasis in thoracic esophageal carcinoma. Chin J Surg 2003, 41:523-525.

10. Fang WT, Chen WH, Chen Y, Jiang Y: Selective three-field lymphadenectomy for thoracic esophageal squamous carcinoma. Dis Esophagus 2007, 20:206-211.

11. Nakagawa S, Kanda T, Kosugi S, Ohashi M, Suzuki T, Hatakeyama K Recurrence pattern of squamous cell carcinoma of the thoracic esophagus after extended radical esophagectomy with three-field lymphadenectomy. J Am Coll Surg 2004, 198:205-211.

doi:10.1186/s13019-014-0150-4

Cite this article as: Guo et al: Clinical study on postoperative recurrence in patients with pNO esophageal squamous cell carcinoma. Journal of Cardiothoracic Surgery 2014 9:150.

\section{Submit your next manuscript to BioMed Central and take full advantage of:}

- Convenient online submission

- Thorough peer review

- No space constraints or color figure charges

- Immediate publication on acceptance

- Inclusion in PubMed, CAS, Scopus and Google Scholar

- Research which is freely available for redistribution 